\title{
Bacterial blight on Sansevieria cylindrica caused by Pseudomonas sp.
}

\author{
Okhee Choi ${ }^{1} \cdot$ Yeyeong Lee ${ }^{2} \cdot$ Byeongsam Kang ${ }^{3} \cdot$ Seunghoe $\mathrm{Kim}^{2} \cdot \mathrm{Jinwoo} \mathrm{Kim}^{1,2}$ (1)
}

Received: 21 May 2021 / Accepted: 11 August 2021 / Published online: 19 August 2021

(c) Australasian Plant Pathology Society Inc. 2021

\begin{abstract}
The African spear or cylindrical snake plant (Sansevieria cylindrica) is commonly used as an ornamental indoor succulent plant. In May 2019, we observed bacterial blight in S. cylindrica grown for interior decoration and air purification in Jinju, South Korea. These symptoms eventually led to leaf desiccation. Similar leaf blight symptoms have been frequently documented on social media; however, as an imported species, the pathogens of $S$. cylindrica have not been reported in South Korea. Based on pathogenicity tests; levan production, oxidase production, pectinolytic activity, arginine dihydrolase production, and tobacco hypersensibility tests; analytical profile index API tests; and phylogenetic analysis based on multilocus sequence analysis using the $16 \mathrm{~S}$ rRNA, $g y r B$, $r p o D$, and $r p o B$ gene sequences, we identified the causative pathogen as Pseudomonas sp. This study is the first to report leaf blight caused by Pseudomonas sp. on S. cylindrica.
\end{abstract}

Keywords Bacterial blight $\cdot$ Multi-locus sequence analysis $\cdot$ Pseudomonas sp. $\cdot$ Sansevieria cylindrica

The African spear or cylindrical snake plant (Sansevieria cylindrica; family Asparagaceae) is a succulent native to Africa. S. cylindrica is a popular indoor ornamental plant due to its long, striped, greenish-gray subcylindrical leaves; it also exhibits strong air purification effects and is easy to cultivate (Wolverton et al. 1989; Takawira and Nordal 2003). In Korea, potted Sansevieria plants are often presented as gifts at opening ceremonies and other auspicious events, and it has been used as a traditional medicine for various diseases in Africa (Tanveer et al. 2017). Sansevieria species have been investigated for their pharmacological activity, producing antimicrobial, antioxidant, antitumor, and antidiabetic effects (Da Silva et al. 2003). The chemical constituents of $S$. cylindrica include steroids, flavonoids, saponins, tannins, and phenolic acids (Said et al. 2015; Tanveer et al. 2017).

Okhee Choi and Yeyeong Lee contributed equally to this work.

Jinwoo Kim

jinwoo@gnu.ac.kr

1 Institute of Agriculture and Life Science, Gyeongsang National University, Jinju 52828, South Korea

2 Department of Plant Medicine, Gyeongsang National University, Jinju, South Korea

3 Division of Applied Life Science, Gyeongsang National University, Jinju, South Korea
In May 2019, leaf blight was observed in S. cylindrica plants used for interior decoration and air purification in Jinju, South Korea. Similarly infected plants were easily found in commercial shops growing $S$. cylindrica for decoration; these were eventually discarded. Many individuals who grow S. cylindrica indoors have tried to determine the cause of its leaf blight by uploading photographs on social media. Because little is known about the pathogens associated with S. cylindrica in Korea, the aim of this study was to isolate and identify the causative agent of leaf blight on S. cylindrica.

Symptoms on S. cylindrica begin from the bottom of the leaf and progress to the tip, eventually leading to severe desiccation of the entire leaf (Fig. 1a). To isolate the pathogen from infected plants, we cut tissue Sects. $(0.5 \mathrm{~cm} \times 0.5 \mathrm{~cm})$ including lesions and healthy areas of leaves using a scalpel, and then the sections were surface-sterilized with $1 \%$ hypochlorite solution and rinsed with sterilized distilled water. The tissue sections were placed in a $1.5-\mathrm{mL}$ microtube containing sterilized distilled water, crushed with a pipette tip, soaked for $10 \mathrm{~min}$, and then mixed evenly. We serially diluted $100 \mu \mathrm{L}$ of bacterial suspension and then spread it onto a $0.5 \times$ trypticase soy agar (TSA) plate. The plates were incubated at $28^{\circ} \mathrm{C}$ for 2 days, and the resulting colonies were re-streaked onto a new TSA plate. We tested 20 isolated colonies for hypersensitivity reaction (HR) in tobacco leaves. Three HR-inducing bacteria (MHGNU B109a-c) 
Fig. 1 Symptoms of bacterial blight caused by Pseudomonas sp. on Sansevieria cylindrica

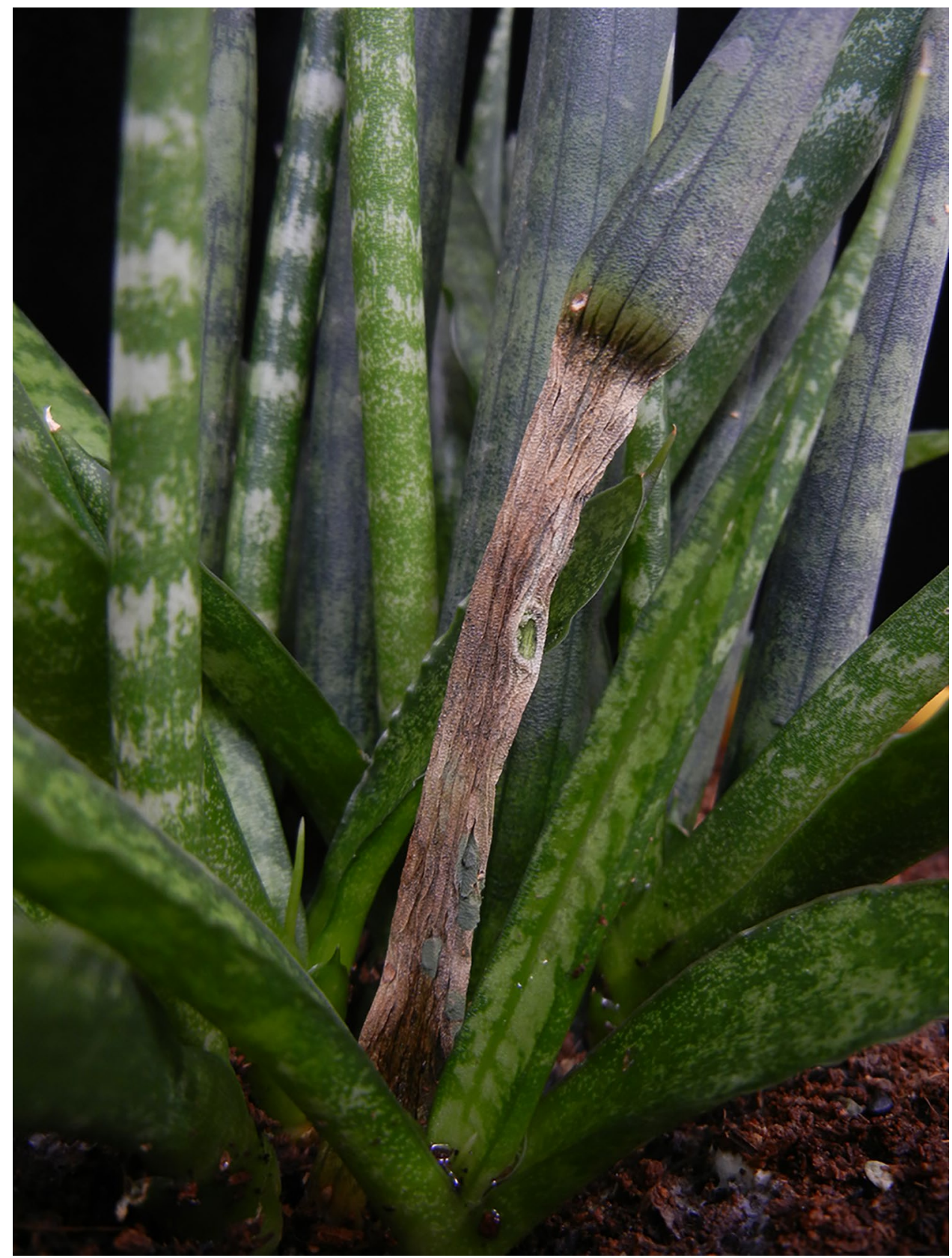

were selected and subjected to levan production, oxidase production, pectinolytic activity, arginine dihydrolase production, and tobacco hypersensibility (LOPAT) tests (Ewing et al. 1960; Klement et al. 1964; Schaad et al. 2001). The three isolates were found to be positive for oxidase production and negative for levan and arginine dihydrolase production and pectinolytic activity. The physiological and biochemical characteristics of the bacterial isolates were tested using the analytical profile index (API) 20NE system (bioMérieux, Marcy l'Etoile, France). All three isolates utilized D-glucose, L-arabinose, D-mannose, D-mannitol, potassium gluconate, capric acid, malic acid, and trisodium citrate, and did not utilize N-acetyl-glucosamine, D-maltose, adipic acid, or phenylacetic acid. All three isolates were negative for the production of indole, urease, $\beta$-glucosidase, protease, and $\beta$-galactosidase. Nitrate was not reduced to nitrite. All three isolates showed non-fluorescence on King's medium B. All three isolates exhibited the same traits in LOPAT and API tests, suggesting that they are the same bacterial species. To confirm the identity of the bacterial pathogens, we analyzed the 16S rRNA gene sequences of the three isolates. Total DNA extraction and polymerase chain reaction amplification were performed as previously described (Choi et al. 2017) using the primers $27 \mathrm{mF}$ (5'-AGAGTTTGATCMTGGCTC AG-3') and 1492mR (5'-GGYTACCTTGTTACGACTT-3') (Lane 1991). The 16S rRNA gene sequences (1388 bp) of 
the three isolates were identical, and that of MHGNU B109a was deposited into GenBank (accession no. MW617234). The DNA sequences were analyzed using the BLASTN program and compared with sequences in the National Center for Biotechnology Information GenBank database. The $16 \mathrm{~S}$ rRNA gene sequence of the isolate was $99.93 \%$ homologous with Pseudomonas sp. strain p34 from Quercus pyrenaica rhizosphere in Spain (Lasa et al. 2019). A representative isolate, MHGNU B109a, was deposited into the Korea Agricultural Culture Collection (KACC 21450).

For phylogenetic analysis, we amplified three housekeeping genes, $g y r B$, rpoD, and rpoB, of KACC 21450 with the following primer sets: UP-1E (5'-CAGGAAACA GCTATGACCAYGSNGGNGGNAARTTYRA-3') and APrU (5'-TGTAAAACGACGGCCAGTGCNGGRTCY TTYTCYTGRCA-3') (Hall et al. 2016); PsEG30F (5'ATYGAAATCGCCAARCG-3') and PsEG790R (5'-CGG TTGATKTCCTTGA-3') (Mulet et al. 2009); and LAPSs (5'-TGGCCGAGAACCAGTTCCGCGT-3') and LAPs27 (5'-CGGCTTCGTCCAGCTTGTTCAG-3') (Tayeb et al. 2005), respectively. The resulting sequences of the $\mathrm{gyr} B$ (920 bp), rpoD (687 bp), and rpoB (1074 bp) genes were deposited into GenBank (accession nos. MW721596, MW727275, and MW727274, respectively). Phylogenetic analysis based on multi-locus sequence analysis (MLSA) and comparison with sequences in the Pseudomonas aeruginosa Metabolome Database (http://genome.ppws.vt. edu/cgi-bin/MLST/home.pl) were performed as described previously (Mulet et al. 2009). A phylogenetic tree based on the concatenated nucleotide sequences of the 16S rRNA gene and three housekeeping genes was generated using the MEGA 7.0 software and the maximum likelihood method (Kumar et al. 2016). The phylogenetic trees based on MLSA showed that KACC 21450 was closely clustered with Pseudomonas abietaniphila (Fig. 2). However, we did not identify KACC 21450 as $P$. abietaniphila because this isolate exhibits different phenotypes than the P. abietaniphila type strain DSM 17554T, being positive for the utilization of D-maltose and the production of urease and arginine dihydrolase (Saati-Santamaría et al. 2018). Further study is required to determine the species of this pathogen.

The pathogenicity test was carried out as described previously (Choi et al. 2013). Leaves of commercially purchased S. cylindrica were disinfected with $70 \%$ ethanol, and $100 \mu \mathrm{L}$ of bacterial suspension $\left(\sim 10^{8} \mathrm{CFU} / \mathrm{ml}\right)$ of KACC 21450 in sterilized distilled water was injected into three leaves of $S$. cylindrica using a syringe. Sterile distilled water was used for negative control inoculation. The inoculated plants were placed in a greenhouse at $25^{\circ} \mathrm{C}$ for 10 days and then evaluated for disease symptoms. In the pathogenicity tests, water soaking symptoms appeared on inoculated leaves 3-4 days after inoculation. The diseased lesions became large and rapidly dried up and either broke or took on a mummified

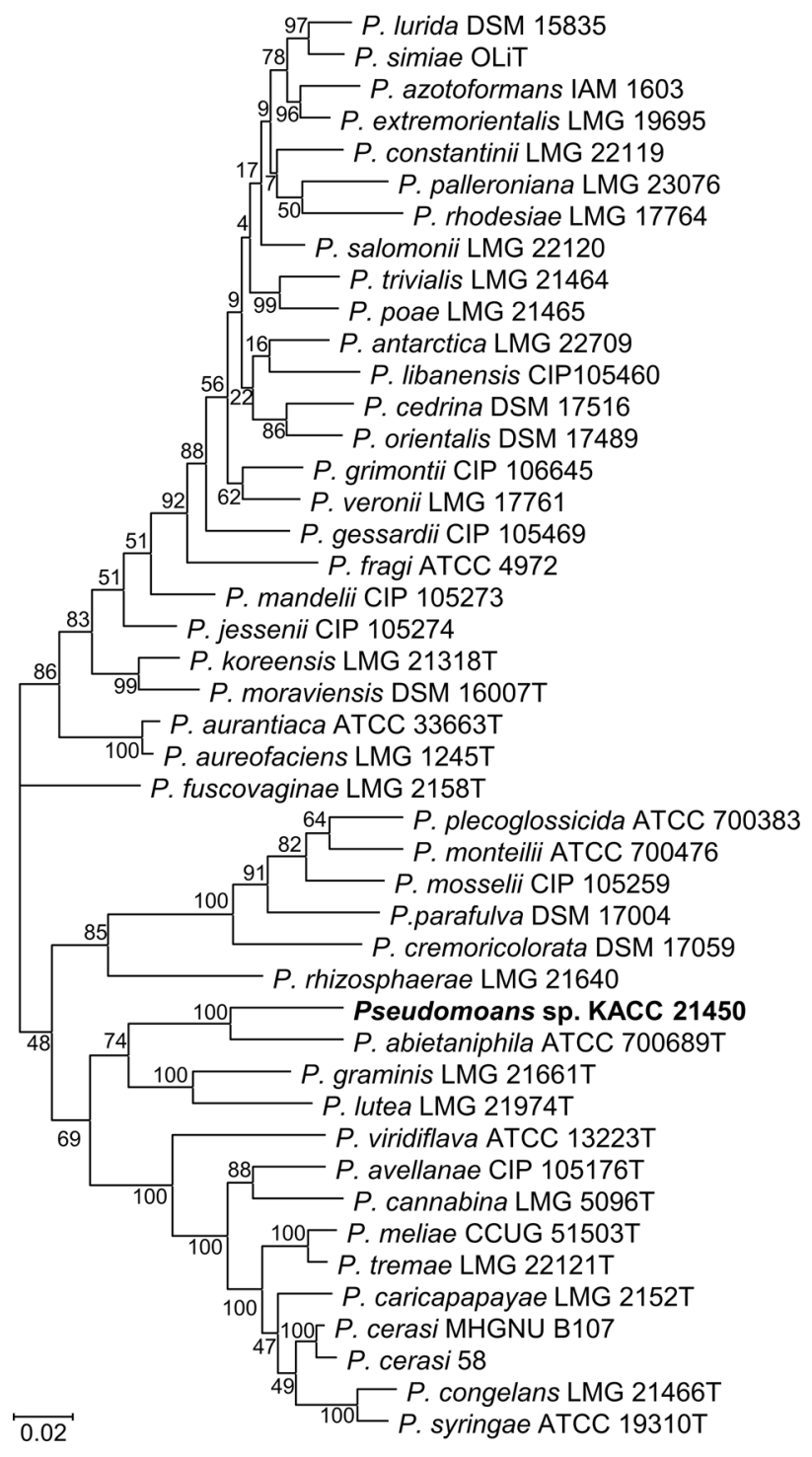

Fig. 2 Phylogenetic tree of Pseudomonas species obtained using the maximum likelihood method based on four concatenated genes (16S rRNA, gyrB, rpoD, and rpoB). The percentages of trees in which associated taxa were clustered together are shown next to the branches. The tree is drawn to scale, with branch lengths measured in numbers of substitutions per site. Bars indicate numbers of nucleotide substitutions per site. The isolate used in the present study is indicated in bold

appearance (Fig. 3a and b). These symptoms were similar to those observed in natural infection. No symptoms were observed on healthy control plants inoculated with sterile distilled water (Fig. 3c and d). To fulfill Koch's postulates, the bacteria were re-isolated from the artificially induced lesions to confirm the 16S rRNA gene sequence.

Thus, the causative bacterial pathogen from $S$. cylindrica was identified as Pseudomonas sp. based on physiological and molecular analyses and pathogenicity tests. To our knowledge, this study is the first to report leaf blight caused 

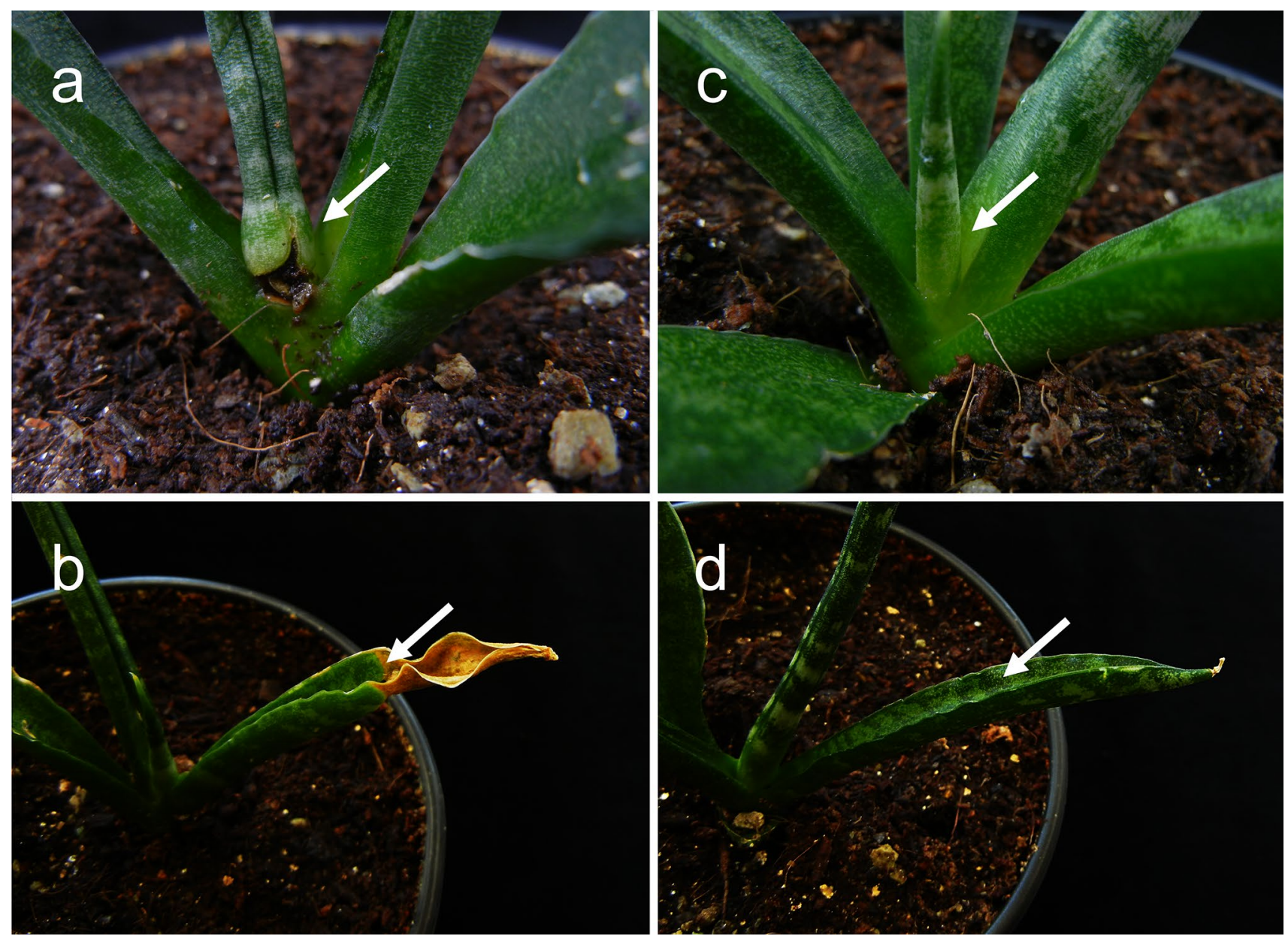

Fig. 3 Pathogenicity tests. (a, b) Symptoms induced by artificial inoculation of KACC 21450 on Sansevieria cylindrica. (c, d) Negative control. Photos were taken 7 and 12 days after inoculation in subcylindrical and noncylindrical leaves, respectively. Arrows indicate inoculation sites

by Pseudomonas sp. on S. cylindrica. These results provide important information for commercial growers of $S$. cylindrica, to improve production and survival of this popular indoor air-purifying and decorative plant.

Acknowledgements This research was supported by Basic Science Research Program through the National Research Foundation of Korea (NRF) funded by the Ministry of Education of the Republic of Korea (2015R1A6A1A03031413).

\section{References}

Ait Tayeb L, Ageron E, Girimont F, Grimont PA (2005) Molecular phylogeny of the genus Pseudomonas based on rpoB sequences and application for the identification of isolates. Res Microbiol 156:763-773

Choi O, Kang B, Cho SK, Park J, Lee Y, Kim W-I, Marunga J, Hwang I, Kim J (2017) Identification of Pseudomonas syringae pv. syringae causing bacterial leaf blight of Miscanthus sinensis. J Plant Dis Prot 124:97-100

Choi O, Kim J (2013) Pantoea stewartii causing stewart's wilt on Dracaena sanderiana in Korea. J Phytopathol 161:578-581
Da Silva AA, Da Silva BP, Parente JP, Valente AP (2003) A new bioactive steroidal saponin from Sansevieria cylindrica. Phytother Res 17:179-182

Ewing JH, Johnson JG (1960) The differentiation of Aeromonas and C27 cultures from Enterobacteriaceae. Syst Evol Microbiol 10:223-230

Hall SJ, Dry IB, Blanchard CL, Whitelaw-Weckert MA (2016) Phylogenetic relationships of Pseudomonas syringae pv. syringae isolates associated with bacterial inflorescence rot in grapevine. Plant Dis 100:607-616

Klement Z, Farkas GL, Lovreicovich L (1964) Hypersensitive reaction induced by phytopathogenic bacteria in the tobacco leaf. Phytopathology 54:474-477

Kumar S, Stecher G, Tamura K (2016) MEGA7: molecular evolutionary genetics analysis version 7.0 for bigger datasets. Mol Bio Evol 33:1870-1874

Lane DJ (1991) 16S/23S rRNA sequencing. In: Stackebrandt E, Goodfellow M (eds) Nucleic acid techniques in bacterial systematics. John Wiley \& Sons, New York, NY, USA, pp 115-147

Lasa AV, Mašínová T, Baldrian P, Fernández-López M (2019) Bacteria from the endosphere and rhizosphere of Quercus spp. use mainly cell wall-associated enzymes to decompose organic matter. PLoS One 14:e0214422 
Mulet M, Bennasar A, Lalucat J, García-Valdé E (2009) An rpoD-based PCR procedure for the identification of Pseudomonas species and for their detection in environmental samples. Mol Cell Probes 23:140-147

Saati-Santamaría Z, López-Mondéjar R, Jiménez-Gómez A, DíezMéndez A, Větrovský T, Igual JM, Velázquez E, Kolarik M, Rivas R, García-Fraile P (2018) Discovery of phloeophagus beetles as a source of Pseudomonas strains that produce potentially new bioactive substances and description of Pseudomonas bohemica sp. nov. Front Microbiol 9:913

Said A, Aboutabl EA, Melek FR, Jaleel GARA, Raslan M (2015) Steroidal saponins and homoisoflavanone from the aerial parts of Sansevieria cylindrica Bojer ex Hook. Phytochem Lett 12:113-118
Schaad NW, Jones JB, Chun W (2001) Laboratory guide for identification of plant pathogenic bacteria. APS Press, Minnesota, USA, p 373

Takawira R, Nordal I (2003) The genus of Sansevieria (family Dracaenaceae) in Zimbabwe. Acta Hortic 552:189-199

Tanveer A, Devendra SN, Faheem KM (2017) Phytochemical analysis, total phenolic content, antioxidant and antidiabetic activity of Sansevieria cylindrica leaves extract. J Nat Prod Resour 3:134-136

Wolverton BC, Anne J, Keith B (1989) Interior landscape plants for indoor air pollution abatement. Final Report-September, 1989, NASA John C. Stennis Space Center 